\title{
Modification of the Drain Current on a Metal-Oxide-Semiconductor Field-Effect Transistor with Ferrite Gate Oxide
}

\author{
N. Wakiya, H. Wada, K. Shimizu, S. Mizukami, M. Machi, N. Mizutani, and K. Shinozaki \\ Department of Metallurgy and Ceramics Science, Tokyo Institute of Technology, \\ 2-12-1-S7-6 O-okayama, Meguro-ku, Tokyo 152-8550, Japan
}

$\left(\mathrm{Mn}_{0.24} \mathrm{Zn}_{0.09} \mathrm{Fe}_{0.67}\right) \mathrm{Fe}_{2} \mathrm{O}_{4}$ (MZF) thin films were grown on Si substrates without and with MOSFETs by pulsed laser deposition (PLD). The deposition of MZF films was carried out by the following two procedures: (1) "1-step deposition," in which 300-nm-thick MZF film was deposited at $800^{\circ} \mathrm{C}$, and (2) "2-step deposition," in which 10-nm-thick MZF film was deposited at $800^{\circ} \mathrm{C}$ and 300 -nm-thick MZF film was deposited at $27^{\circ} \mathrm{C}$ followed by post-deposition annealing at $800^{\circ} \mathrm{C}$ for $1 \mathrm{~h}$ under an $\mathrm{O}_{2}$ pressure of $1.3 \mathrm{x}$ $10^{-3} \mathrm{~Pa}$. For both preparation procedures, epitaxial growth of MZF thin film with (111)-orientation was observed. The saturation and remanent magnetization of MZF thin film depends on the preparation procedure, and the highest magnetization was obtained for film prepared by 1-step deposition. A microfabrication process in which preparation of MZF thin film was limited to the gate areas of Si-MOSFETs was attempted, using a combination of the abovementioned processes and etching processes: (1) 1-step deposition followed by wet chemical etching, and (2) 2-step deposition. In the 2-step deposition, a lift-off process was inserted before $27^{\circ} \mathrm{C}$ deposition. $I_{D^{-}} V_{D S}$ characteristics before and after magnetization of the MZF thin film was measured. As a result, modification of the drain current was observed. The modification was brought about by the Lorentz force, which was caused by the magnetic field induced by remanent magnetization. For MZF/Si-MOSFETs fabricated by 1-step deposition, the modification of the drain current was on the order of several $\mu \mathrm{A}$ when the gate voltage was $5 \mathrm{~V}$. For MZF/Si-MOSFETs fabricated by 2-step deposition, the modification of the drain current was on the order of a few dozen pA when the gate voltage was $0.8 \mathrm{~V}$. It was also clarified that the degree of modification increases with decreasing gate length. These results suggest that this principle could be used in a novel nonvolatile memory application.

Key words: metal-oxide-semiconductor field-effect transistor, modification of drain current, ferrite thin film, fabrication process, epitaxial growth, novel magnetic recording

\section{Introduction}

The effect of an external magnetic field on metal-oxide-semiconductor field-effect transistors (MOSFETs) is currently known as MAGFETs ${ }^{1-4)}$. However, MAGFETs are magnetic sensors using the lateral Hall effect. We have proposed a concept of drain current $\left(I_{\mathrm{D}}\right)$ modification in MOSFETs using the Lorentz force from an external magnetic field and a magnetic field induced by remanent magnetization ${ }^{5,6}$. It was found that the magnetic field increased the $I_{\mathrm{D}}$ when the carriers were pushed away from the interface, and that a magnetic field with the opposite direction increased $I_{\mathrm{D}}$ slightly. This phenomenon was related to the potential change and carrier mobility ${ }^{6}$. The carrier mobility in the channel of MOSFETs is approximately related to the scattering by bulk and surface phonons, Coulomb scattering, and surface roughness scattering ${ }^{7}$. Therefore, these scattering factors decrease the mobility near the interface, and the scattering is less in the bulk. We expect that this concept will be useful in the design of novel memory devices. The principle of such a memory device is schematically shown in Fig. 1. In the configuration, ferromagnetic thin film is deposited on the gate areas of MOSFETs. An external magnetic field is applied to the ferromagnetic layer, and is then removed. The ferromagnetic layer is then magnetized to generate a magnetic field induced by remanent magnetization. The magnetic field exerts a Lorentz force on the carriers in the channels of MOSFETs.

The purpose of this work is to establish microfabrication of MZF thin film on the gate areas of Si-MOSFETs, and to examine the degree of drain current modification generated by the principle. The effect of the gate length was also examinedin order to increase the modification of $I_{\mathrm{D}}$.

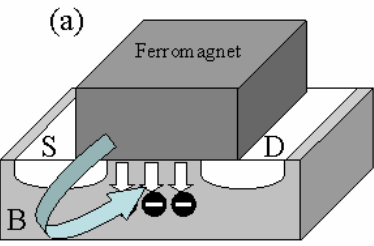

Downward Lorentz force

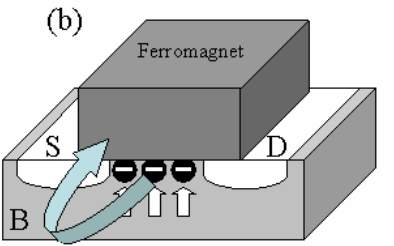

Upward Lorentz force
Fig. 1 Schematic drawing of the principle of the new memory: an external magnetic field is first applied to magnetize the ferromagnetic layer, and is removed.

(a) A downward Lorentz force, which is caused by the magnetic field induced by the remanent magnetization, pushes the carriers in the channel into the bulk to increase mobility.

(b) An upward Lorentz force pushes the carriers toward the interface to decrease mobility. 


\section{Experimental method}

$\left(\mathrm{Mn}_{0.24} \mathrm{Zn}_{0.09} \mathrm{Fe}_{0.67}\right) \mathrm{Fe}_{2} \mathrm{O}_{4}$ (MZF) thin films were grown on $\mathrm{Si}$ substrates with and without MOSFETs by pulsed laser deposition (PLD). The deposition of MZF thin films was carried out by the following two procedures:

(1) “One-step deposition," in which 300-nm-thick MZF films were deposited at $800^{\circ} \mathrm{C}$ in $1.3 \mathrm{~Pa} \mathrm{O}_{2}$. Prior to the deposition of the MZF thin film, 8-nm-thick yttria-stabilized zirconia (YSZ, $800^{\circ} \mathrm{C}$ in $\left.7.3 \times 10^{-2} \mathrm{~Pa} \mathrm{O}_{2}\right), 6$-nm-thick $\mathrm{CeO}_{2}\left(800^{\circ} \mathrm{C}\right.$ in $7.3 \times 10^{-2}$ $\left.\mathrm{Pa} \mathrm{O}_{2}\right)$ and 8 -nm-thick $\mathrm{ZnCo}_{2} \mathrm{O}_{4}\left(600^{\circ} \mathrm{C}\right.$ in $\left.1.3 \mathrm{~Pa} \mathrm{O}_{2}\right)$ layers were deposited. The YSZ layer was essential for realizing epitaxial oxide film preparation on $\mathrm{Si}(001)$ substrate $^{8)}$. The $\mathrm{CeO}_{2}$ layer was introduced to improve crystallinity. $\mathrm{ZnCo}_{2} \mathrm{O}_{4}$ was used to improve the magnetic properties ${ }^{6}$.

(2) "Two-step deposition," in which 10-nm-thick MZF film was deposited at $800^{\circ} \mathrm{C}$ in $1.3 \mathrm{~Pa} \mathrm{O}_{2}$, cooled to $27^{\circ} \mathrm{C}$ and $300-n m-t h i c k$ MZF thin film was deposited at $27^{\circ} \mathrm{C}$ in $1.3 \mathrm{~Pa} \mathrm{O}_{2}$. Prior to the deposition of the MZF thin film, a 5-nm-thick YSZ thin layer was deposited. Since this process was rather complicated, deposition of $\mathrm{CeO}_{2}$ and $\mathrm{ZnCo}_{2} \mathrm{O}_{4}$ layers was not carried out. The 2-step deposition films were annealed at $800^{\circ} \mathrm{C}$ for $1 \mathrm{~h}$ in $1.3 \times 10^{-3} \mathrm{~Pa} \mathrm{O}_{2}$.

$\mathrm{X}$-ray diffraction analysis, in-situ reflection high-energy electron diffraction (RHHED) observation, and $M-H$ hysteresis curve measurements using a vibrating sample magnetometer (VSM) were carried out for MZF thin films deposited on $\mathrm{Si}$ substrates without MOSFETs.

Microfabrication of MZF thin film on the gate areas of Si-MOSFETs was also carried out. Two kinds of microfabrication were attempted:

[1] Wet chemical etching

[2] Lift-off process

These two methods are related to the above two preparation procedures.

Wet chemical etching was carried out after 1-step deposition was finished. We found that $\mathrm{YSZ}$ and $\mathrm{CeO}_{2}$ films are insoluble in $32 \% \mathrm{HCl}$ solution, while $\mathrm{ZnCo}_{2} \mathrm{O}_{4}$ and $\mathrm{MZF}$ films are soluble. By using the difference in solubility, removal of MZF/ZC films except for the gate areas was carried out by photolithography and wet chemical etching. No annealing was carried out after wet chemical etching. A lift-off process was introduced after 10-nm-thick MZF film was deposited at $800^{\circ} \mathrm{C}$ and cooled to $27^{\circ} \mathrm{C}$. At $27^{\circ} \mathrm{C}$, lift-off resist (LOR) was coated on the surface of $\mathrm{Si}$ substrate with MOSFETs except for the gate area. 300 -nm-thick MZF film was deposited at $27^{\circ} \mathrm{C}$ in $1.3 \mathrm{~Pa} \mathrm{O}_{2}$, and the LOR was then removed. Subsequently, microfabrication of MZF/YSZ thin film on the gate area was carried out. Finally, the film was annealed at $800^{\circ} \mathrm{C}$ for $1 \mathrm{~h}$ in $1.3 \times 10^{-3} \mathrm{~Pa} \mathrm{O}_{2}$.

After processes [1] and [2], Pt electrodes were fabricated on the pads of source, gate, and drains by dc magnetron sputtering at $27^{\circ} \mathrm{C}$ followed by a lift-off process. Figure 2 shows schematic cross-sections and laser microscope photographs of MOSFETs after these processes.
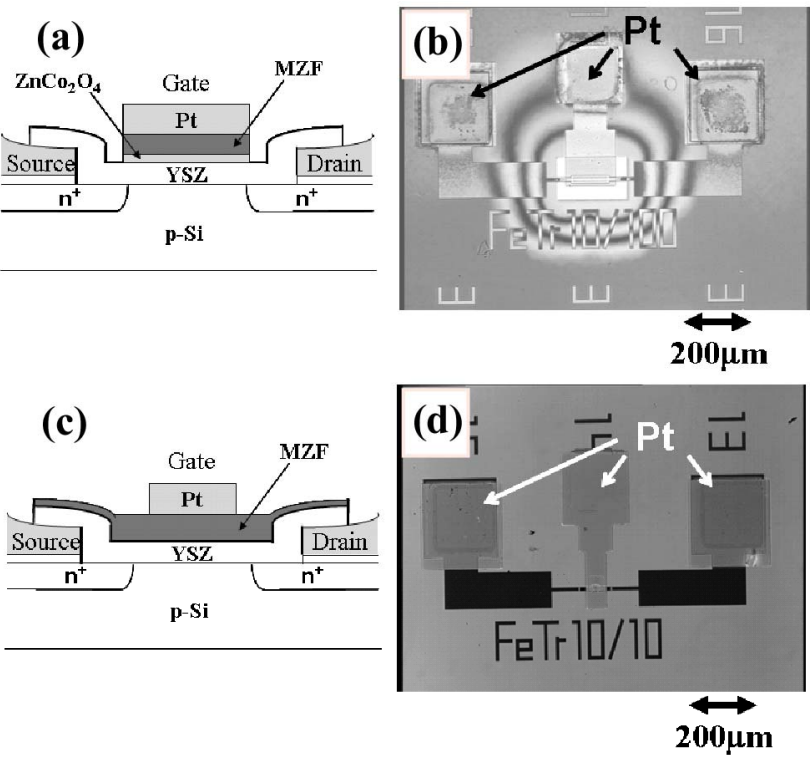

Fig. 2 Schematic cross-section of MOSFETs with MZF thin film on the gate area fabricated by (a) 1-step deposition followed by a wet chemical etching process and (c) 2-step deposition followed by a lift-off process. (b) and (d) show laser microscope images after corresponding processes.

The Si substrates with MOSFETs were specially ordered ones (n-channel enhancement type with a gate width $(W)$ of 10 $\mu \mathrm{m}$, and gate lengths $(L)$ of $0.8,1,2,5,10,100$, and $300 \mu \mathrm{m}$.). The $I_{\mathrm{D}}-V_{\mathrm{DS}}$ characteristics of the MOSFETs were measured by using a pA meter. The thickness of the film was determined by using both a surface profile meter and X-ray fluorescent spectroscopy.

\section{Results and discussion}

X-ray diffraction results indicate that the MZF films deposited on $\mathrm{Si}(001)$ by both 1- and 2-step depositions show (111)-orientation. Figure 3(a) shows an in-situ RHEED image of 60-nm-thick-MZF thin film deposited at $800^{\circ} \mathrm{C}$ by 1 -step deposition. A streak pattern shows that the film is epitaxially grown. Figure 3(b) shows a (100) X-ray pole figure image of MZF thin film deposited at $800^{\circ} \mathrm{C}$ by 1 -step deposition. The observed twelve poles indicate that MZF thin film is epitaxially grown. However, the epitaxial growth consists of four crystallographic domains of MZF coexisting with every $30^{\circ}$ 
rotation along the $\phi$ axis, because only three poles are expected to be observed every $120^{\circ}$ for a single-crystallographic-domain epitaxial MZF(111) thin film. Figures 3(c) and 3(d) show in-situ RHEED images of 10-nm-thick MZF thin films deposited at $800^{\circ} \mathrm{C}$ and $300-\mathrm{nm}$-thick MZF thin film deposited on (c) at $27^{\circ} \mathrm{C}$. Though the RHEED pattern shown in Fig. 3(d) is rather diffuse, a streaky pattern was also observed. This means that epitaxial growth continued even after $27^{\circ} \mathrm{C}$ deposition. Actually, the $\mathrm{X}$-ray pole figure pattern after 2-step deposition was the same as that after 1-step deposition, shown in Fig. 3(b). Such low-temperature epitaxial growth of nickel zinc ferrite (NZF) and MZF has also been reported in the literature ${ }^{9)}$.
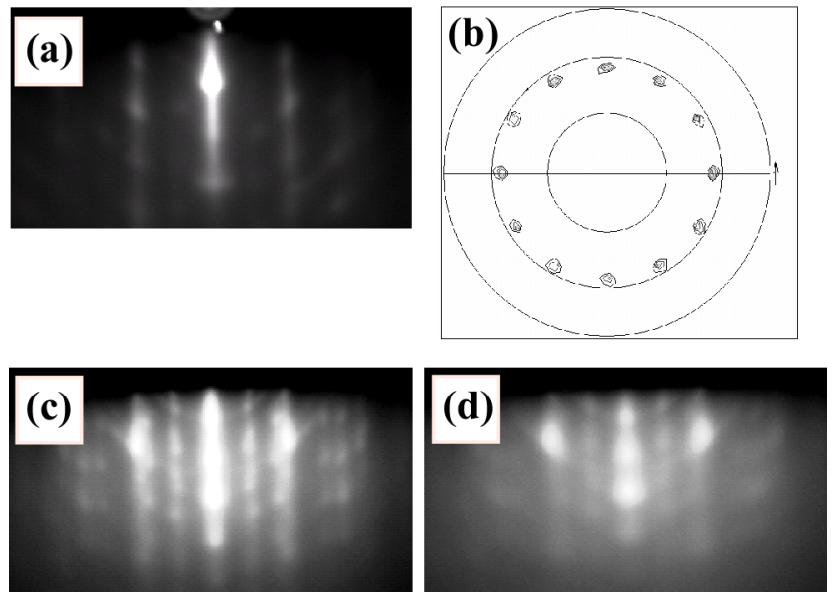

Fig. 3 RHEED image of (a) 60-nm-thick MZF thin film deposited at $800^{\circ} \mathrm{C}$ (1-step deposition). (b) a (100) X-ray pole figure image of MZF thin film deposited at $800^{\circ} \mathrm{C}$ (1-step deposition). RHEED images of (c) 10-nm-thick MZF thin film deposited at $800^{\circ} \mathrm{C}$ and (d) 300-nm-thick MZF thin film deposited on (c) at $27^{\circ} \mathrm{C}$ (2-step deposition).
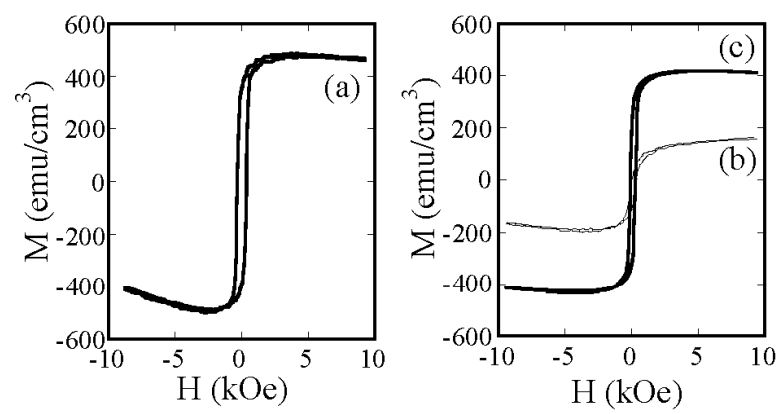

Fig. $4 \quad M-H$ hysteresis curves of MZF thin film prepared by (a) 1-step deposition and (b) and (c) 2-step deposition before and after annealing at $800^{\circ} \mathrm{C}$, respectively.

Figure 4(a) shows room-temperature $M-H$ hysteresis curves of
MZF thin film deposited by 1-step deposition. This film exhibited a remanent magnetization of $370 \mathrm{emu} / \mathrm{cm}^{3}$. Figures 4(b) and 4(c) show room-temperature $M-H$ hysteresis curves of MZF thin film deposited by 2-step deposition before and after annealing at $800^{\circ} \mathrm{C}$ for $1 \mathrm{~h}$. This figure shows that the remanent magnetization of as-deposited 2-step deposition MZF thin film before and after annealing was 50 and $260 \mathrm{emu} / \mathrm{cm}^{3}$, respectively.

Figure 5(a) shows the $I_{D^{-}} V_{D S}$ characteristics of MZF/Si-MOSFETs thin film fabricated by 1-step deposition followed by wet chemical etching. The film was as-deposited before application of an external magnetic field. For this MOSFET, the gate length $L$ and gate width $W$ were 5 and $10 \mu \mathrm{m}$, respectively, and the gate voltage $V_{G}$ was $5 \mathrm{~V}$. In this work, an external magnetic field of $0.44 \mathrm{~T}$ was applied and removed. Application of an external magnetic field causes remanent magnetization for the MZF thin film, and a magnetic field was induced by the remanent magnetization. It should be noted that the direction of the magnetic field induced by the remanent
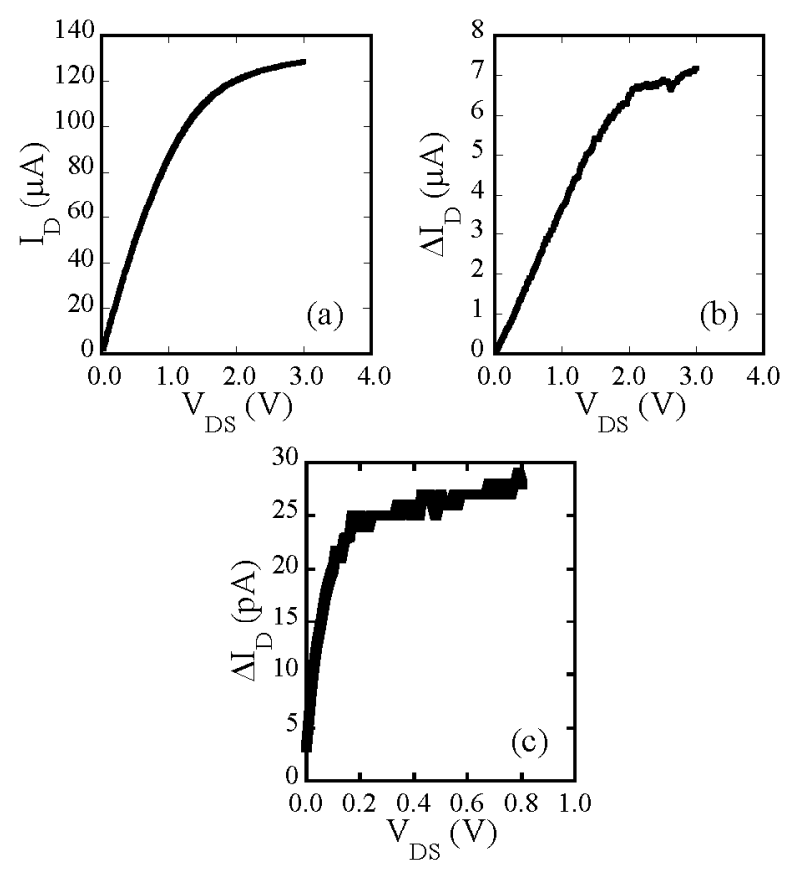

Fig. 5 (a) $I_{D}-V_{D S}$ characteristics of MZF/Si-MOSFET thin film before application of a magnetic field $\left(L=5 \mu \mathrm{m}, W=10 \mu \mathrm{m}, V_{\mathrm{G}}\right.$ $=5 \mathrm{~V})$.

Change in $\Delta I_{\mathrm{D}}$ with $V_{\mathrm{DS}}$ after (b) 1-step deposition followed by wet chemical etching ( $\left.L=5 \mu \mathrm{m}, W=10 \mu \mathrm{m}, V_{\mathrm{G}}=5 \mathrm{~V}\right)$, and (c) 2-step deposition with a lift-off process $(L=10 \mu \mathrm{m}, W=10 \mu \mathrm{m}$, $V_{\mathrm{G}}=0.8 \mathrm{~V}$ ), where $\Delta I_{D}$ is $I_{D}$ (after magnetic field application) $-I_{D}$ (before magnetic field application)). 
Magnetization is opposite to that of the original external magnetic field. In this work, the direction of the magnetic field (induced by remanent magnetization) was selected so as to generate a downward Lorentz force, as shown in Fig. 1(a). Figure $5(\mathrm{~b})$ shows the degree of modification of the drain current $\left(\Delta I_{D}\right) \quad\left(\Delta I_{D}=I_{D}\right.$ (after magnetic field application) $I_{D}$ (before magnetic field application)) with $V_{D S}$. The value of $\Delta I_{D}$ was on the order of several $\mu \mathrm{A}$. Figure 5(c) shows the relationship between $\triangle I_{D}$ and $V_{D S}$ of MZF/Si-MOSFETs thin film fabricated by using a combination of 2-step deposition and a lift-off process. For this MOSFET, the gate length $L$ and gate width $W$ were 10 and $10 \mu \mathrm{m}$, respectively, and the gate voltage $V_{G}$ was $0.8 \mathrm{~V}$. In this case, the $\Delta I_{D}$ was on the order of a few dozen pA. In this figure, a $\Delta I_{D}$ value of $3 \mathrm{pA}$ was observed when $V_{D S}=0 \mathrm{~V}$. This finite $\Delta I_{D}$ could be ascribed to the leakage current of MOSFETs at $V_{D S}=0 \mathrm{~V}$ and the effect of measurement error. Due to the low process yield, it was difficult to compare $I_{D}-V_{D S}$ characteristics using the same $L$ and $V_{G}$ for both processes. The difference in the order of $\Delta I_{D}$ is likely to contain several factors such as $L, V_{G}$, and process factors. To reduce the number of factors, the change in $\Delta I_{D}$ with gate length was examined in an external magnetic field of $0.4 \mathrm{~T}$ (Fig. 6). Figure 6 shows the change in $\Delta I_{\mathrm{D}}$ with $L$. For this experiment, the gate width $W$ was $10 \mu \mathrm{m}, V_{G}$ and $V_{D S}$ were 3 and $4 \mathrm{~V}$, respectively, and B was 0.4 $\mathrm{T}$. This figure clearly shows that $\Delta I_{D}$ increases with decreasing $L$. The results of this work suggest that ferromagnetic thin film fabricated on the gate area of Si-MOSFET could modify the drain current, and that the effect is enhanced by small gate length and high gate voltage.

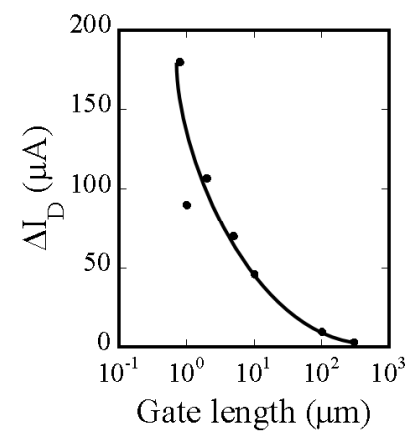

Fig. 6 Change in $\Delta I_{\mathrm{D}}$ with gate length for an Si-MOSFET $\left(W=10 \mu \mathrm{m}, V_{\mathrm{G}}=3 \mathrm{~V}, V_{\mathrm{DS}}=4 \mathrm{~V}, B=0.4 \mathrm{~T}\right)$.

\section{Conclusions}

Modification of the drain current of an Si-MOSFET by a magnetic field induced by remanent magnetization of ferromagnetic thin film was investigated. As the ferromagnetic thin film, $\left(\mathrm{Mn}_{0.24} \mathrm{Zn}_{0.09} \mathrm{Fe}_{0.67}\right) \mathrm{Fe}_{2} \mathrm{O}_{4}$ (MZF) was grown on $\mathrm{Si}$ substrates with and without MOSFETs by pulsed laser deposition (PLD). The deposition of MZF films was carried out by following two procedures: (1) 1-step deposition, in which 300-nm-thick MZF film was deposited at $800^{\circ} \mathrm{C}$, and (2) 2-step deposition, in which 10-nm-thick MZF film was deposited at $800^{\circ} \mathrm{C}$ and 300 -nm-thick MZF film was deposited at $27^{\circ} \mathrm{C}$ followed by post-deposition annealing. For both preparation procedures, epitaxial growth of MZF thin film with (111)-orientation was observed. The saturation and remanent magnetization of MZF thin film depends on the preparation procedure, and the highest magnetization was obtained for film prepared by 1-step deposition. Microfabrication of MZF thin films in which preparation of MZF thin film was limited to the gate areas of Si-MOSFETs was attempted, using a by the combination of the abovementioned processes and etching processes: (1) 1-step deposition followed by wet chemical etching, and (2) 2-step deposition. In the 2-step deposition, a lift-off process was inserted before $27^{\circ} \mathrm{C}$ deposition. The $I_{D^{-}} V_{D S}$ characteristics before and after magnetization of MZF thin film was measured. As a result, modification of the drain current was observed. The modification was brought about by the Lorentz force, which was caused by the magnetic field induced by remanent magnetization. For MZF/Si-MOSFETs fabricated by 1-step deposition, the modification of the drain current was on the order of several $\mu \mathrm{A}$ when the gate voltage was $5 \mathrm{~V}$. For MZF/Si-MOSFETs fabricated by 2-step deposition, the modification of the drain current was on the order of a few dozen $\mathrm{pA}$ when the gate voltage was $0.8 \mathrm{~V}$. It was also clarified that the degree of modification increases with decreasing gate length. These results suggest that this principle could be used ina novel nonvolatile memory application.

\section{References}

1) G. R. M. Rao and W. N. Carr: Solid State Electronics, 14, 995 (1971).

2) R. Agrawal, P. K. Yadava, R. Dwivedi, and S. K. Srivastava: Sensors and Actuators A, 28, 21 (1991).

3) D. R. Briglio, A. Nathan, and H. P. Baltes: Can. J. Phys., 65, 842 (1987).

4) N. Mathieu, P. Giordano, and A. Chovet: Sensors and Actuators A, 32, 656 (1992).

5) N. Wakiya, K. Shinozaki, and N. Mizutani: Jpn. J. Appl. Phys., 41, 7242 (2002).

6) N. Wakiya, K. Shimizu, S. Mizukami, K. Shinozaki, and N. Mizutani: Appl. Phys. Lett., 85, 3772 (2004).

7) T. Ando, A. B. Fowler, and F. Stern: Rev. Mod. Phys., 54, 437 (1982).

8) N. Wakiya, T. Yamada, K. Shinozaki, and N. Mizutani: Thin Solid Films, 371, 211 (2000).

9) T. Kiyomura and M. Gomi: Jpn. J. Appl. Phys., 40, 118 (2001).

Received Oct. 19, 2005; Revised Nov. 28, 2005; Accepted Dec. 16,2005 